\title{
Power Management Based on Droop Control in DC Microgrid Pu Xie ${ }^{1, a}$, Run-Sheng Yang ${ }^{1, b}$ and Jin-ning Liu $^{1, c}$ \\ ${ }^{1}$ Mechanical Engineering College, Shijiazhuang 050003, China

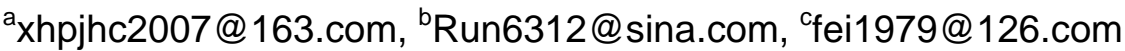

Keywords: power management; DC microgrid; droop control; MATLAB

\begin{abstract}
This paper deals with a DC-micro-grid with renewable energy and conventional energy. In the DC microgrid system, it is a key problem to keep balance between supply power and demand power. Based on the analysis of the operational priority of micro source and load, the priority model of micro source and load is achieved according to actual case. According to DC bus voltage droop control principle, the energy management strategy is formulated. Finally, the effectiveness of the proposed method based on droop control is examined in a MATLAB/Simulink environment.
\end{abstract}

\section{Introduction}

A DC microgrid is consisted of a wide range of power supply sources such as diesel generators, photovoltaic arrays and wind generator. Unlike the power produced by synchronous generators in conventional large-scale power plants, the output power of the distributed power supply is always changing because of the influence of environmental factors[1]. It is a key problem to keep balance between the power supply and the power of user demand. DC microgrid is expected mainly for bidirectional power distribution, bidirectional communication, and reducing mismatching between supply and demand [2]. Some key system variables of the microgrid need to be monitored and adequately controlled in order to achieve the power and engery management.The load power needs to be allocated to different distributed power reasonably to maximize the use of distributed energy in case of the stable operation of the system at the same time.

\section{Configuration of DC microgrid}

The microgrid is supplied by the DC bus because the DC microgrids have more advantages as followings. For the demand side, there are many of a electrical load is possible to be DC fed in efficiency manner without AC/DC conversions [3]. Another reason is DC microgrid is integrated with micro sources such as PV or fuel cells at higher power efficiency. Finally, only the voltage needs to be stabilized in a DC microgrid, so various DC sources can operate together without regarding of phase. Whereas in the AC microgrid requires each element to have almost identical wave shapes in order to combine with each other.

The proposed DC microgrid is composed of a PV, wind power generation system, a battery, DC loads in a DC distribution system and AC loads in a AC distribution system. The battery plays an important role as an energy storage component. The batteries work alternately in the state of charging, discharging or float charging [4,5]. There are both alternative and conventional energy sources in a dc microgrid, so it is important to have an effective power management strategy to achieve efficient system operation.

\section{Power management strategy based on priority model}

Priority model of micro source power supply The operating state of the system is judged according to the energy management strategy, and the output control signal of micro source is given. The priority of micro source is determined according to the environment, resources and other factors. In this strategy, traditional energy sources are used only if the load demand cannot be met by the alternative energy. 
Firstly, the priority of distributed power supply is set up as the priority of $1 \sim \mathrm{N}$ and the priority of storage battery is lower than that of distributed power sources. And the priority of battery absorb power is lower than that of load. According to the priority setting, the system is connected to the distributed power supply, storage battery and diesel generator.

It is can be seen that the priority model of micro grid power supply system is changed by the system state of the system from control flow of the model. The power management system can adjust the system state to make the system from one equilibrium state to another. The number of distributed power supply in the control model can be determined according to the actual situation. It is generally a diesel generator or power grid, and the battery can be changed into other energy storage device. The participation sequence of the energy sources is demonstrated in Fig. 1.

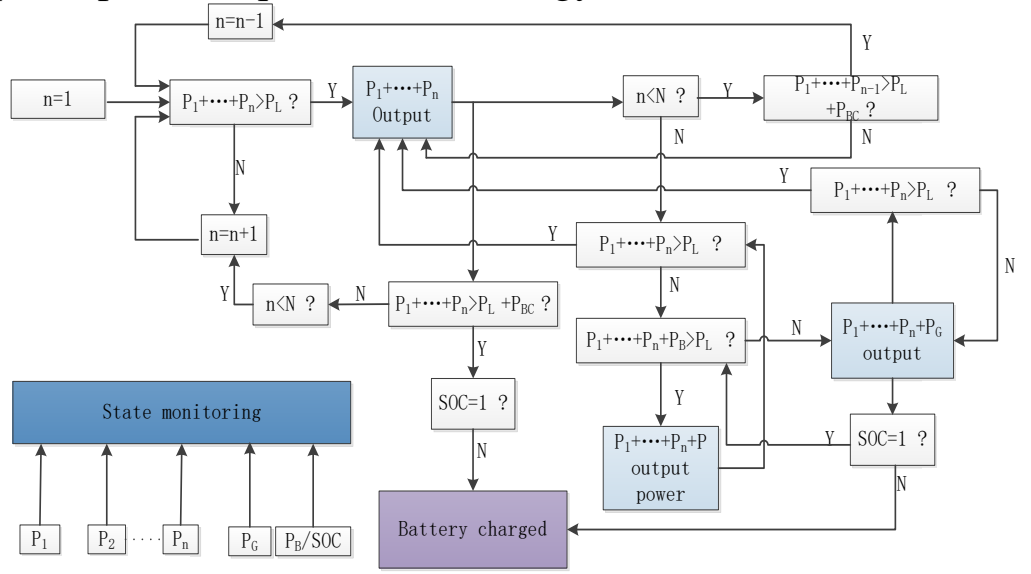

Fig.1 Priority control model of micro-grid power supply

Priority model of loads The DC microgrid cannot provide all the demand power of the load needed when some of the micro sources are failure. At this time, the power supply should be according to the priority of the load. Because the diesel generator and battery power supply can provide the maximum power demand of all the loads, so when the load priority to set the battery to reach the discharge threshold, diesel generator failure or damage cannot continue to power, set the output maximum power at this time, according to the load of the importance from high to low load power set for the establishment of load priority operation model, as shown in figure 2.

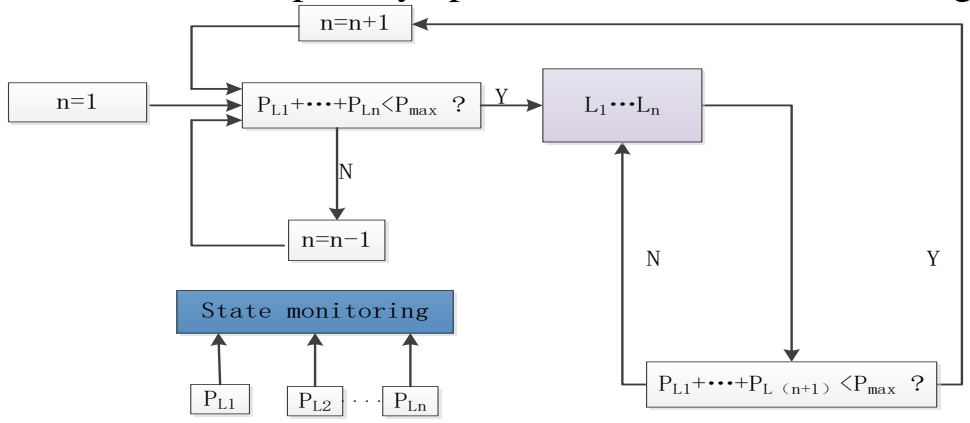

Fig. 2 Priority control model of load operation

DC bus voltage droop control principle Because of the droop characteristics of the DC bus voltage in the DC microgrid system, when the power supply cannot meet the load demand of power supply the output voltage will drop, so the voltage deviation is introduced very meaningful. When the voltage drop is equal to the voltage deviation,which is a threshold value of the power supply. Then according to the priority model of the micro sources, the next priority source is connected in the microgrid system. At this time, the lowest micro sources state is steady voltage power output whereas other distributed sources is by current control of the lowest priority.

The droop control of DC bus voltage is seen in Fig.3. When the first micro source meet the demand of load power requirements, the first micro source is a constant voltage power supply mode, that is the output voltage is $U_{\text {ref1 }}$. When the load power or micro source output power is changed, which resulted in supply power is less than the load demand power then the bus voltage will drop. At this time, the first micro source is working at droop voltage state. When the dc bus voltage drop 
to threshold voltage of the second micro sources $U_{\text {ref2 }}$ the second micro source will be connected at constant voltage power supply mode in the DC microgrid system.

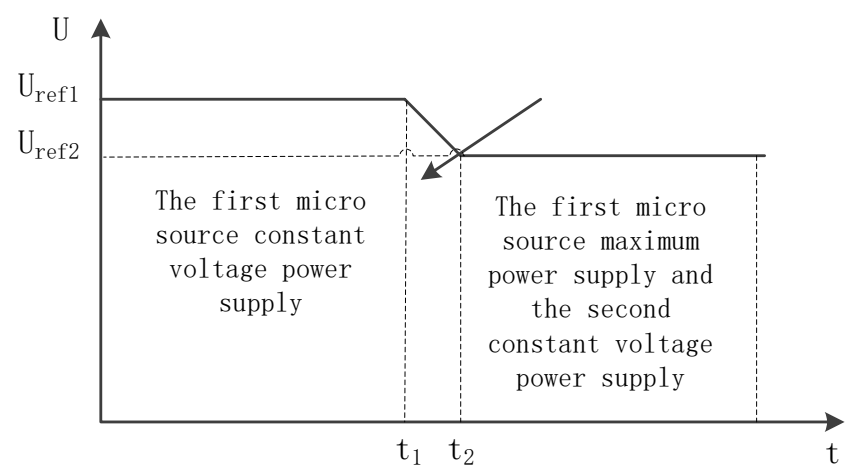

Fig.3 Droop control principle of dc bus voltage

\section{Simulation of micro source coordination control}

Firstly, the power and the battery are connected into the system according to the priority mode, and the control strategy of the power allocation is carried out according to the priority. In order to verify of the power allocation the load is constant, SOC $=40.5 \%$, and the parameters of the wind and optical resource are as follows. The simulation result can be seen from Fig.4.

t: $0-1 \mathrm{~s}, \mathrm{~T}=24^{\circ} \mathrm{C}, \mathrm{S}=50 \mathrm{~W} / \mathrm{m}^{2}, \mathrm{v}=7 \mathrm{~m} / \mathrm{s} ;$
t: $1-2 \mathrm{~s}, \mathrm{~T}=25^{\circ} \mathrm{C}, \mathrm{S}=800 \mathrm{~W} / \mathrm{m}^{2}, \mathrm{v}=6 \mathrm{~m} / \mathrm{s} ;$
t: $2-3 \mathrm{~s}, \mathrm{~T}=24^{\circ} \mathrm{C}, \mathrm{S}=400 \mathrm{~W} / \mathrm{m}^{2}, \mathrm{v}=5 \mathrm{~m} / \mathrm{s} ;$
t: $3-4 \mathrm{~s}, \mathrm{~T}=25^{\circ} \mathrm{C}, \mathrm{S}=100 \mathrm{~W} / \mathrm{m}^{2}, \mathrm{v}=4 \mathrm{~m} / \mathrm{s} ;$

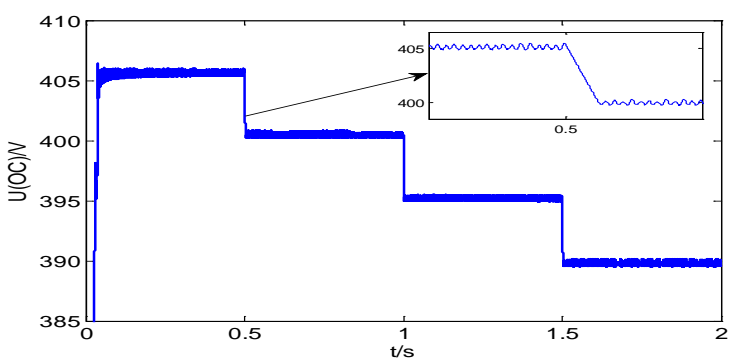

Fig.4-1 DC bus voltage

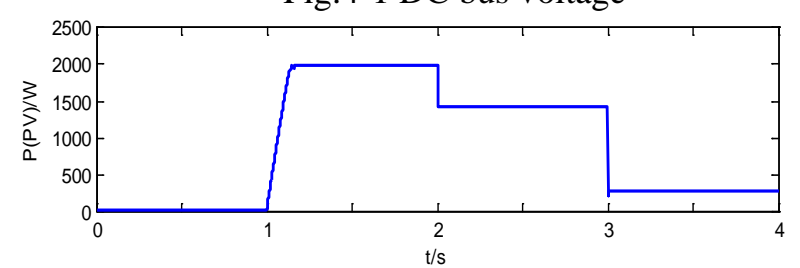

Fig.4-3 Output power of PV

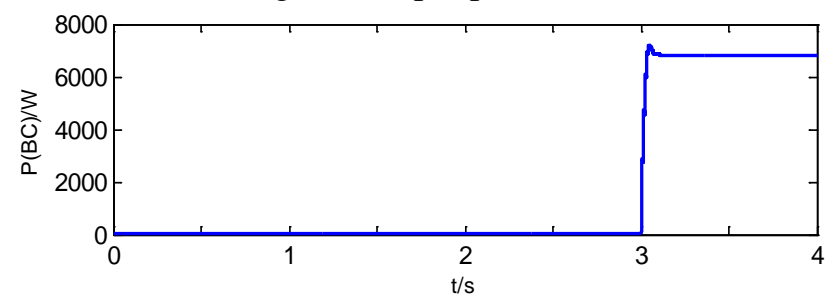

Fig.4-5 Charge power of storage battery

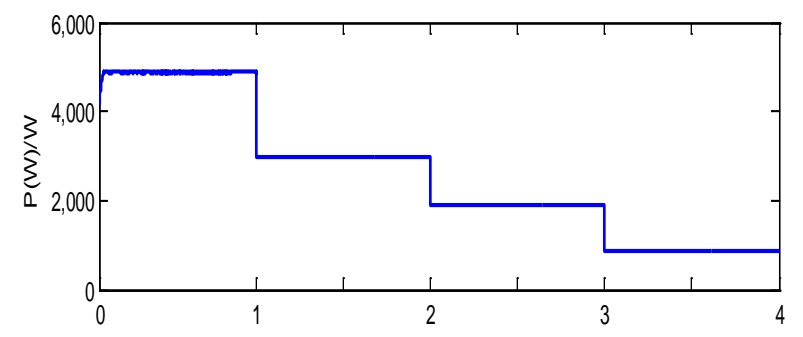

Fig4-2 Output pow㪀r of wind generating

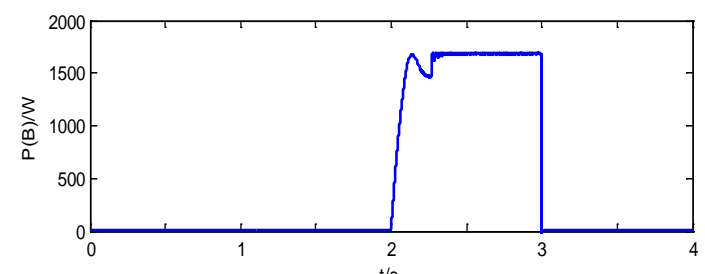

Fig.4-4 Discharge power of storage battery

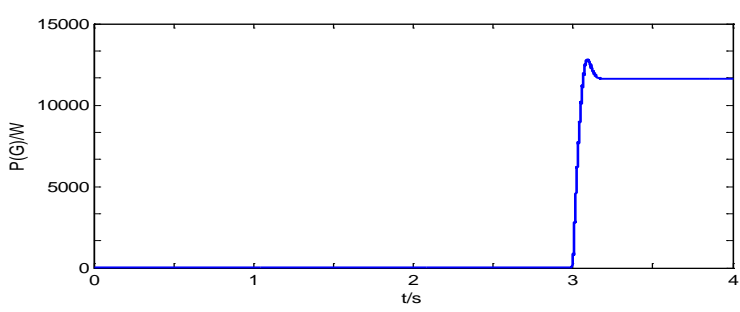

Fig.4-6 Output power of diesel generator

$\mathrm{t}=0 \sim 1 \mathrm{~s}$, according to the calculation of wind power generator output maximum power is $5 \mathrm{KW}$. The wind power generator power supply can meet the load demand power. Photovoltaic unit is not connected to the microgrid, and the microgrid works at the first mode. Voltage of dc bus is $405 \mathrm{~V}$. 
$\mathrm{t}=1 \mathrm{~s}$, the wind speed decreases, the output power is less than the load power demand. The bus voltage drops. The maximum power output of wind generator is $3.2 \mathrm{KW}$, the output power of PV is $2 \mathrm{KW}$, the battery cannot be charged. The work mode of microgrid is switched from mode one to mode two. The voltage of dc bus voltage is $400 \mathrm{~V}$.

$t=2 s$, the output power of the wind turbine and photovoltaic cell is reduced and cannot meet the load power demand, the voltage of dc bus drops to 395V.Then the battery is connected to the microgrid system and the wind turbine and photovoltaic battery is at maximum power output state. The work mode of microgrid is switched from mode two to mode five. The voltage of dc bus voltage is $395 \mathrm{~V}$.

$\mathrm{t}=3 \mathrm{~s}$, the output power is reduced and the battery reaches the discharge termination threshold. The supply power of microgrid cannot meet the load demand power. When the voltage of dc bus is drop down to $390 \mathrm{~V}$ the diesel generator is connected to the microgrid. The battery is charged at constant current of $40 \mathrm{~A}$ because the remaining power is greater than the maximum battery charge current. The work mode of microgrid is switched from mode five to mode six. The voltage of dc bus voltage is $390 \mathrm{~V}$.

The simulation verified that the dc microgrid system can manage the micro sources according to the priority mode when the wind and light resources varies. The highest priority of micro source can achieve the maximum power output. The fluctuation of the bus voltage is decreased so that the dc microgrid system is always in the power balance between supply and demand.

\section{Summary}

This paper presents stable power supply strategies for a DC microgrid system and stable operation strategies based on droop control of bus voltage. Based on the analysis of the operational priority of micro source and load, the priority model of micro source and load is achieved according to actual case. According to DC bus voltage droop control principle, the energy management strategy is formulated. Finally, the simulation is examined in a MATLAB/Simulink environment verified that the dc microgrid system can manage the micro sources according to the priority mode when the wind and light resources varies.

\section{Acknowledgements}

This paper is supported by National Natural Science Foundation of China (No. 51307184).

\section{References}

[1] Sao CK, Lehn PW. Control and power management of converter fed microgrids. IEEE Trans Power Syst 2008;23(3):1088-98.

[2] Thyagarajan K, Davari A, Feliachi A. Load sharing control in distributed generation system. In: Proceedings of the 37th southeastern symposium on system theory (SSST), Tuskegee; March 2005. p. 424-8.

[3] Katsprakakis DA, Paradakis N, Kozirakis G, Minadakis Y, Christakis D, Kondaxakis K. Electricity supply on the island of Dia based on renewable energy sources (R.E. S). Applied Energy 2009;86:516-27.

[4] Jiayi Huang, Chuanwen Jiang, Rong Xu. A review on distributed energy resources and MicroGrid. Renewable and Sustainable Energy Reviews 2008;12 (9):2472-83.

[5] $\mathrm{Hu}$ Weihao, Chen Zhe, Wang Yue, Wang Zhaoan. Wind power fluctuations mitigation by DC-link voltage control of variable speed wind turbines. IEEE Transactions on Power Electronics 2008;10(4):108-16. 